\title{
Characterization of fusion plasmas in the cylindrical DTU inertial electrostatic confinement device
}

Rasmussen, J.; Jensen, T.; Korsholm, S.B.; Kihm, N. E.; Ohms, F.K.; Gockenbach, M.; Schmidt, B.S.; Goss, E.

Published in:

Physics of Plasmas

Link to article, DOI:

$10.1063 / 5.0013013$

Publication date:

2020

Document Version

Publisher's PDF, also known as Version of record

Link back to DTU Orbit

Citation (APA):

Rasmussen, J., Jensen, T., Korsholm, S. B., Kihm, N. E., Ohms, F. K., Gockenbach, M., Schmidt, B. S., \& Goss, E. (2020). Characterization of fusion plasmas in the cylindrical DTU inertial electrostatic confinement device. Physics of Plasmas, 27(8), [0012924]. https://doi.org/10.1063/5.0013013

\section{General rights}

Copyright and moral rights for the publications made accessible in the public portal are retained by the authors and/or other copyright owners and it is a condition of accessing publications that users recognise and abide by the legal requirements associated with these rights.

- Users may download and print one copy of any publication from the public portal for the purpose of private study or research.

- You may not further distribute the material or use it for any profit-making activity or commercial gain

- You may freely distribute the URL identifying the publication in the public portal 


\section{Characterization of fusion plasmas in the cylindrical DTU inertial electrostatic confinement device}

Cite as: Phys. Plasmas 27, 083515 (2020); https://doi.org/10.1063/5.0013013

Submitted: 07 May 2020 . Accepted: 21 July 2020 . Published Online: 06 August 2020

(iD J. Rasmussen, (iD T. Jensen, (DD S. B. Korsholm, (D) N. E. Kihm, F. K. Ohms, M. Gockenbach, B. S. Schmidt, and (iD) E. Goss
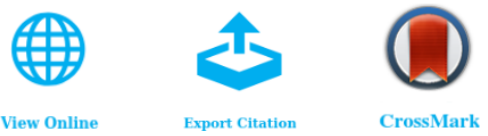

View Online

Export Citation

\section{ARTICLES YOU MAY BE INTERESTED IN}

Laser-plasma instabilities in long scale-length plasmas relevant to shock-ignition

Physics of Plasmas 27, 082704 (2020); https://doi.org/10.1063/5.0010920

Review of pulsed power-driven high energy density physics research on Z at Sandia

Physics of Plasmas 27, 070501 (2020); https://doi.org/10.1063/5.0007476

Validation of heat transport modeling using directly driven beryllium spheres

Physics of Plasmas 27, 082701 (2020); https://doi.org/10.1063/5.0005776

\section{AIP Advances Fluids and Plasmas Collection}




\title{
Characterization of fusion plasmas in the cylindrical DTU inertial electrostatic confinement device
}

Cite as: Phys. Plasmas 27, 083515 (2020); doi: 10.1063/5.0013013

Submitted: 7 May 2020 - Accepted: 21 July 2020 .

Published Online: 6 August 2020

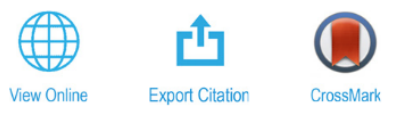

\author{
J. Rasmussen, ${ }^{\text {a) }}$ (D) T. Jensen, (iD S. B. Korsholm, (iD N. E. Kihm, (D) F. K. Ohms, M. Gockenbach, B. S. Schmidt, \\ and E. Goss iD
}

\author{
AFFILIATIONS \\ Technical University of Denmark, Fysikvej 309, Lyngby, Denmark \\ ${ }^{a}$ Author to whom correspondence should be addressed: jeras@fysik.dtu.dk
}

\begin{abstract}
Inertial electrostatic confinement offers a relatively simple and cost-effective means of generating fusion plasmas for research and industrial applications. Here, we present the experimental setup and discharge characteristics of the inertial electrostatic confinement device at the Dept. of Physics, Technical Univ. of Denmark. Special features of this setup include a cylindrical anode and the novel use of 3D printed soccerball-like cathode grids of different sizes. Measurements with these grids show $25 \%$ higher fusion neutron rates than with manually manufactured grids with larger wire spacings. Additionally, we observe significantly higher neutron rates with smaller grids, with spherical rather than cylindrical cathodes, and when using the vacuum chamber, rather than a second spherical grid, as the anode. Ion-orbit simulations predict a core density in the ion distribution in good agreement with optical measurements, confirming that asymmetries in the cathode grid potential prevent a fully convergent ion flow. The simulations also demonstrate that the asymmetry of the electric field induced by the voltage stalk lowers the characteristic ion recirculation by a factor of four, and we discuss measures to circumvent this. Comparing measurements and simulations conducted with a spherical and cylindrical grid reveals tentative evidence that fusion reactivity is highly corelocalized, pointing to ion-neutral fusion as the dominant reaction. We also quantify the thermionic and impact-induced secondary electron emission in the device, showing that only the latter can potentially suppress the ion current during normal operation.
\end{abstract}

Published under license by AIP Publishing. https://doi.org/10.1063/5.0013013

\section{INTRODUCTION}

An Inertial Electrostatic Confinement (henceforth IEC) device is an example of a device capable of generating and confining a nuclear fusion plasma. In its simplest form, a so-called "fusor," an IEC device consists of a vacuum chamber with a gas at low pressure and a metal grid at the center. A large negative voltage is applied to this grid, which then acts as a cathode in an electrode, with the anode represented by a second, larger grid or by the vacuum chamber walls. Positively charged ions in the ambient gas will accelerate through the deep electrostatic potential well toward the center of the chamber. Inside the cathode grid, the density and energy of ions can become high enough for fusion to occur. While simple fusor configurations are not suitable for net energy production, ${ }^{1,2}$ they remain a cost-effective means of generating plasma and fusion neutrons for research and industrial applications. Particular advantages relative to magnetic confinement fusion include the simplicity of the engineering design, the relative ease with which ions can be accelerated to high energies relevant to a particular fusion reaction, and the possibility to study advanced fusion fuels such as $\mathrm{D}-{ }^{3} \mathrm{He}$ and $\mathrm{p}-{ }^{11} \mathrm{~B}$.

A common IEC configuration comprises a spherical vacuum chamber, with a spherical cathode grid nested concentrically inside a larger anode grid. However, contributions to the development of IEC devices and the study of electrostatically confined plasmas have also come from setups involving cylindrical vacuum chambers. ${ }^{3-5}$ Particle interactions in all these devices comprise a complex interplay of scattering, collisions, and charge exchange reactions in a slightly anisotropic background electric field, as determined by the overall geometry of the setup and of the externally applied conditions. The discharge characteristics and fusion reactivity can, therefore, be highly devicedependent and must be characterized in each experiment in order to assess the dominant physical processes and to optimize fusion rates.

Since 2017, the Dept. of Physics at the Technical Univ. of Denmark (DTU) has operated a cylindrical gridded DC glow-discharge IEC device. Unlike the case for a number of other university-run 
cylindrical IEC experiments, this is a single-grid setup with the vacuum chamber acting as the electrically grounded anode during normal operation. This maximizes the electrode distance allowed by the chamber, and also implies that the ion and electron velocity distributions might be anisotropic and that a range of inter-electrode distances may be of relevance for generating and sustaining plasma breakdown in the device.

Here, we describe the DTU IEC device and present the first measurements and Monte Carlo-based modeling results of the particle dynamics and fusion reactivity in the fusor. Of particular interest is the dependence on the geometry and relative size of the cathode and anode, which has returned different results in a number of previous experiments. ${ }^{2,4,6-9}$ As part of this effort, we have developed and tested a series of 3D-printed soccerball-shaped cathode grids, aimed at improving ion convergence and recirculation in the device. We also focus on identifying items for optimization of the setup, on the dominant fusion reactants, and on differences in fusion reactivity with respect to other similar devices. Addressing these issues represents a first step toward a more targeted optimization of discharge performance and neutron production in our fusor and may help to inform similar effort at other experiments.

In Sec. II, we describe the experimental setup and the modeling performed in the present work, and Sec. III presents the results on discharge characteristics, particle dynamics, and fusion rates in the device as characterized by measurements and simulations. We discuss some of these results and their implications for the spatial distribution of fusion reactivity in Sec. IV, before concluding with a summary in Sec. V. For convenience, all charges and electric potentials are considered as positive throughout.

\section{SETUP AND MEASUREMENTS}

\section{A. Experimental setup and operation}

The DTU fusor consists of a grounded cylindrical vacuum chamber, in which a cathode grid is suspended. A negative voltage is supplied to this grid by a $U=80 \mathrm{kV}, I=25 \mathrm{~mA}$ Glassman LT-XT power supply delivering a maximum DC power of $2 \mathrm{~kW}$. As the high-voltage feedthrough is dimensioned to $U \leq 60 \mathrm{kV}$ only, this is the maximum voltage applied in normal operation. The fusor is operated in the constant-current mode, in which the input current limits the discharge voltage. A serially connected $R=71 \mathrm{k} \Omega$ resistor bank is included in the circuit for improving discharge stability, and all voltages reported in this paper have been corrected for the voltage drop $\Delta U=-I R$ across this resistor. Figure 1 shows a schematic and photo of the setup, and Table I summarizes characteristic parameters of the fusor.

The vacuum chamber has $3.3 \mathrm{~mm}$ thick steel walls, with a cylindrical cap at the top to which the high-voltage feedthrough is attached. Two of the four viewports allow diagnostic access to the plasma through vacuum windows consisting of $3.0 \mathrm{~mm}$ thick Kodial glass (alkali borosilicate 7056) with good x-ray transmission efficiency at photon energies $E \gtrsim 20 \mathrm{keV}$. The grounded vacuum chamber acts as an anode during standard operation. A voltage stalk comprising a $\mathrm{Cu}$ wire enclosed by a quartz tube connects the cathode grid to the power supply. The tube serves to eliminate ion impact on the $\mathrm{Cu}$ wire and to suppress any arc discharges to the nearby vessel walls. At voltages approaching $U=60 \mathrm{kV}$, arcing in the chamber is nevertheless often observed, and discharges tend to be unstable.

Evacuation of the vacuum chamber to a base pressure of $P \approx 1 \times 10^{-3} \mu$ bar using a two-stage pump system allows efficient removal of impurities prior to injection of the desired gas. When evacuated from atmospheric pressure, "baking" of the chamber through
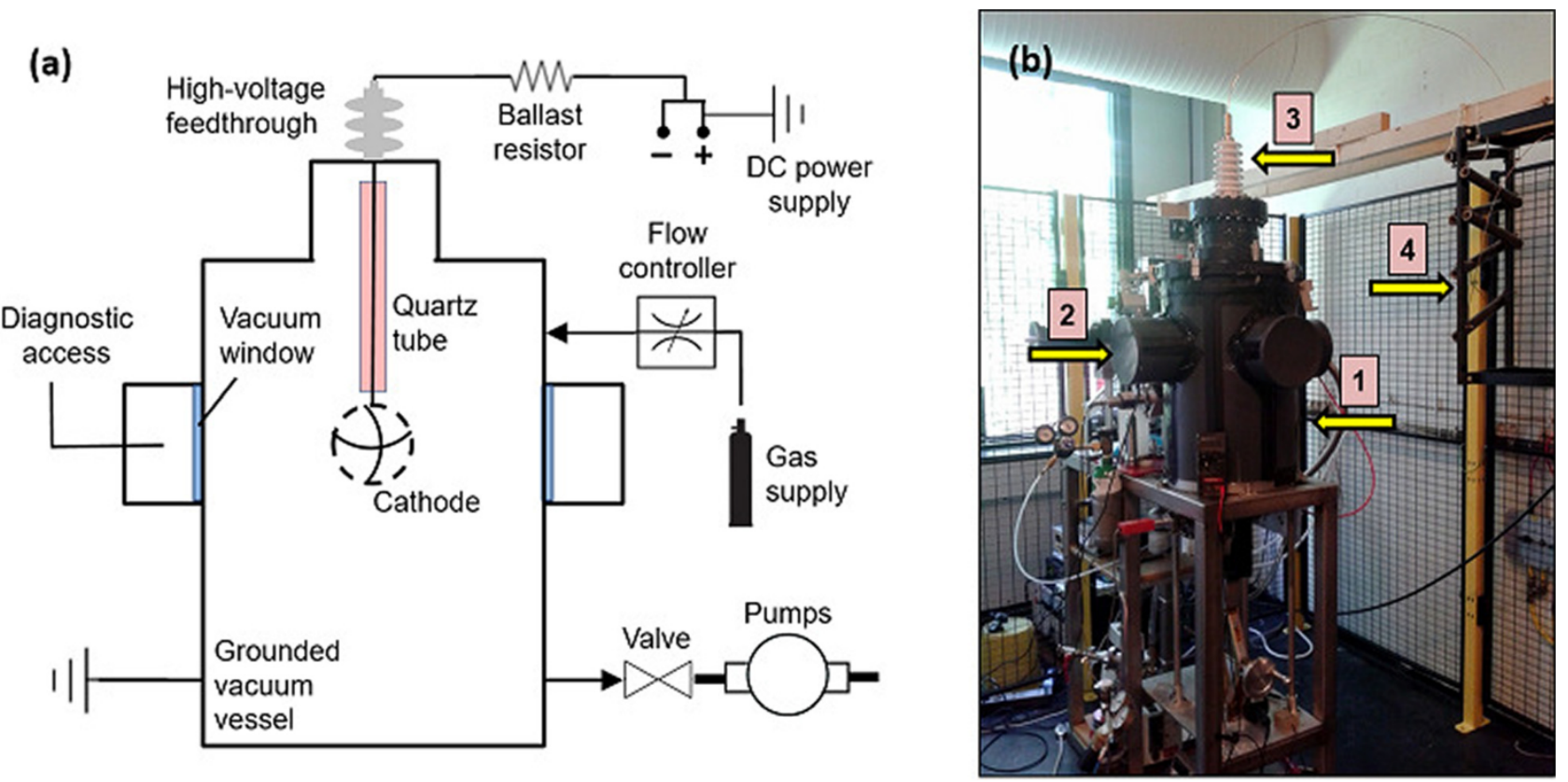

FIG. 1. (a) Schematic of the experimental setup. (b) Photo of the fusor, with key components labeled: 1-Vacuum vessel (anode) with lead cladding. 2-Diagnostic viewport. 3-60 kV high-voltage feedthrough. 4-Resistor bank. 
TABLE I. Dimensions and general properties of the experiment.

\begin{tabular}{lc}
\hline \hline Parameter & Value \\
\hline Anode inner radius & $r_{\mathrm{an}}=17.8 \mathrm{~cm}$ \\
Anode inner height & $h_{\mathrm{an}}=55.5 \mathrm{~cm}$ \\
Anode wall & $3.3 \mathrm{~mm}$ stainless steel \\
Anode total volume & $58 \mathrm{~L}$ \\
Quartz tube inner radius & $7.6 \mathrm{~mm}$ \\
Quartz tube thickness & $0.5 \mathrm{~mm}$ \\
Max. voltage & $U_{\max }=60 \mathrm{kV}$ \\
Max. current & $I_{\max }=25 \mathrm{~mA}$ \\
\hline \hline
\end{tabular}

standard plasma operation for a few tens of minutes is usually necessary to fully remove impurities released by outgassing from the chamber walls. The setup is maintained at roughly constant pressure by bleeding gas into the chamber with the vacuum pump running. The maximum voltage that can be sustained at a given current is observed to decrease linearly with increasing pressure $P$ across the typical operating range of $P \approx 1-10 \mu \mathrm{bar}$. To obtain reproducible discharge conditions, it is, therefore, important to regulate the background chamber pressure, which can otherwise fluctuate due to, e.g., plasma heating and minor variations in the gas flow rate. This control is achieved through a feedback loop based on linear control theory, using flow controllers and an MKS 628 Baratron pressure gauge. This allows the pressure to be stably maintained to a precision of $2 \%$ during longpulse operation. When fusion neutron production is not the main quantity under study, the fusor is often operated with gases other than $\mathrm{D}_{2}$, such as $\mathrm{H}_{2}, \mathrm{He}$, or Ar. In the present work, we will present results obtained with all these gases.

High fusion rates in gridded IEC devices generally rely on effective recirculation of ions through the cathode grid and on good ion core convergence. Experience with a hand-wound spherical tungsten grid, as well as with a corresponding cylindrical grid with the same base radius (geometrically congruent with the anode at $\sim 1 / 5$ its linear dimensions), has shown that plasma stability, grid heating, and fusion performance are sensitive to any geometric irregularities of the grids. Hence, in addition to the above grids, a series of 3D-printed spherical titanium grids with diameter $d=4,8,12$, and $16 \mathrm{~cm}$ are employed in the fusor. These highly isotropic soccer ball-like grids consist of 20
TABLE II. Properties of cathode grids used with the fusor, including wire diameters $d_{\text {wire }}$ and geometrical transparencies $\eta$.

\begin{tabular}{llllll}
\hline \hline Material & Shape & Dimensions & $d_{\text {wire }}$ & Openings & $\eta$ \\
\hline $\mathrm{W}$ & Sphere & $d=8 \mathrm{~cm}$ & $0.7 \mathrm{~mm}$ & 16 & $96 \%$ \\
$\mathrm{~W}$ & Cylinder & $d=8.5 \mathrm{~cm}$ & $0.7 \mathrm{~mm}$ & 16 & $97 \%$ \\
& & $h=11 \mathrm{~cm}$ & & & \\
$\mathrm{Ti}$ & Sphere & $d=4 \mathrm{~cm}$ & $1.0 \mathrm{~mm}$ & 32 & $89 \%$ \\
$\mathrm{Ti}$ & Sphere & $d=8 \mathrm{~cm}$ & $1.0 \mathrm{~mm}$ & 32 & $93 \%$ \\
$\mathrm{Ti}$ & Sphere & $d=12 \mathrm{~cm}$ & $1.0 \mathrm{~mm}$ & 32 & $95 \%$ \\
$\mathrm{Ti}$ & Sphere & $d=16 \mathrm{~cm}$ & $1.0 \mathrm{~mm}$ & 32 & $96 \%$ \\
\hline \hline
\end{tabular}

hexagonal and 12 pentagonal openings, and their fixed $1.0 \mathrm{~mm}$ wire thickness results in high geometric transparencies $\eta$ that differ slightly among the grids. These features set the grids apart from the otherwise somewhat similar buckyball-style grids considered elsewhere. ${ }^{10}$

The purposes of these 3D-printed grids are to improve ion recirculation, as facilitated by geometrically opposite grid openings and the absence of locations with high grid surface density, and to reduce localized grid heating associated with inhomogeneities in the electric field. The grids also enable comparison of the fusion performance for exactly congruent grids of different sizes and transparencies. Finally, their size range allows the smallest of these grids to be inserted inside the largest, such that the latter can act as a spherical anode in place of the vacuum chamber walls. Figure 2 shows examples of the different grid types employed in the fusor, and Table II summarizes their properties. Note that the fractional area $1-\eta$ blocked by the grid wires varies by a factor of $\sim 4$ among the different grids.

The vacuum chamber, including viewport openings, is covered by $2.0 \mathrm{~mm}$ thick lead plates to shield from x rays produced through electron energy losses (see Sec. III D). Measurements with both a Geiger counter and x-ray spectrometer have confirmed our model predictions that no significant $\mathrm{x}$-ray emission can penetrate this shielding for photon energies $E \leq 60 \mathrm{keV}$. The entire experimental setup is located inside an electrically grounded safety cage, with an interlock mechanism that automatically interrupts the power supply if the cage door is opened during operation. An N300-GCA-07W Geiger-Müller counter continuously monitors ionizing radiation outside the cage.
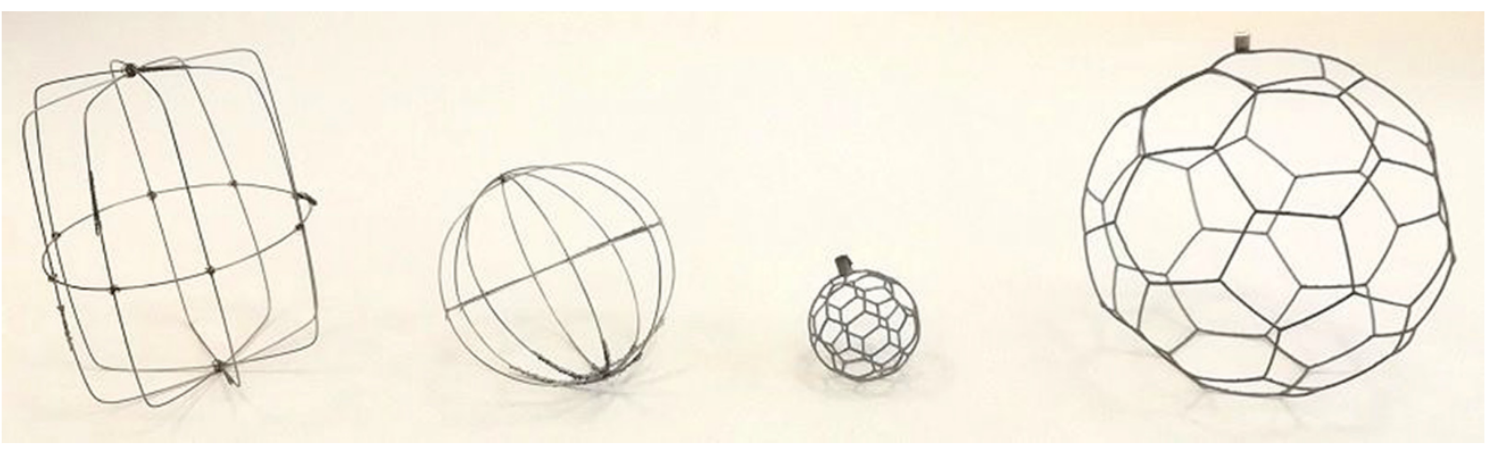

FIG. 2. Selected cathode grids employed in the fusor. From left to right: manually produced cylindrical W grid $(d=8.5 \mathrm{~cm})$; corresponding spherical $W$ grid $(d=8 \mathrm{~cm}) ; 3 \mathrm{D}$ printed Ti grids $(d=4$ and $12 \mathrm{~cm})$. 


\section{B. Diagnostic equipment and modeling}

Diagnostic equipment for monitoring plasma and fusion performance includes a neutron detector, optical, and x-ray spectrometers, and a web camera.

Fusion neutrons from $\mathrm{D}+\mathrm{D} \rightarrow{ }^{3} \mathrm{He}+\mathrm{n}$ are detected using a GS-neutron-150 counter mounted in the immediate vicinity of the vacuum chamber. Neutron transport simulations with the MCNP $\operatorname{code}^{11}$ assuming a simplified spherical device geometry suggest that the vast majority of these neutrons proceed unimpeded through the $3.3 \mathrm{~mm}$ steel wall and $2.0 \mathrm{~mm} \mathrm{~Pb}$ cladding of the vacuum chamber, while largely retaining their birth energy. Moreover, both the steel wall and $\mathrm{Pb}$ shield are predicted by these simulations to act as neutron multipliers, in combination increasing the emerging neutron flux by $15 \%$. This is taken into account in all neutron rate estimates presented below. The neutron detector response to these nearly mono-energetic neutrons has been calibrated using an ${ }^{241} \mathrm{Am}-\mathrm{Be}(\alpha, \mathrm{n})$ source emitting $2-10 \mathrm{MeV}$ neutrons at a rate known to within $\sim 1 \%$.

A Thorlabs CCS100/M optical spectrometer with $0.5 \mathrm{~nm}$ resolution across its $350-700 \mathrm{~nm}$ wavelength range is employed for impurity monitoring and for measurements of Doppler-induced line broadening along different directions in the plasma. The spectrometer views the plasma through optical fibers with $50 \mu \mathrm{m}$ apertures and is wavelength-calibrated using an HgAr lamp. In addition, an AmpTek XR-100 CdTe solid-state $\mathrm{x}$-ray spectrometer with a PX5 pulse processor has been employed for $\mathrm{X}$-ray continuum measurements of free-free and free-bound plasma emission. Finally, a standard web camera monitors the distribution of excited atoms in the chamber and allows identification of various plasma "modes" and of excessive cathode grid heating.

In addition to experimental diagnostics, simulations using COMSOL Multiphysics ${ }^{12}$ v. 5.3 have been carried out in order to help characterize the electric fields and ion orbits in the fusor. These simulations are conducted using the COMSOL Weak Form PDE Interface and the Particle Tracing Module. The modeled geometry of the anode follows the dimensions outlined in Table I and includes a number of small inlets at the bottom of the vacuum chamber left over from previous uses of the chamber. The observation windows are modeled as Pyrex glass to account for their different permittivity. The voltage stalk and quartz tube are also implemented in the model according to Table I, while the cathode grid is modeled to match the dimensions and transparency of the manually produced spherical and cylindrical $\mathrm{W}$ grids listed in Table II.

The particle simulations track 5000 non-interacting deuterons, initialized with zero velocity. For the ion source distribution, we consider two cases that are likely to bracket the true distribution in our experiment: a random uniform distribution within a sphere with the radius equal to the base radius $r=17.8 \mathrm{~cm}$ of the vacuum chamber, and-motivated by other model results ${ }^{13}$ - a case where ionization takes place only in the vicinity of the cathode on a "shell" congruent with the cathode but with linear dimensions 50\% larger. Ions are removed from the simulation upon collision with any surface. The simulations are terminated at $t=1.5 \mu$ s after 638 timesteps of duration $d t=2.35 \mathrm{~ns}$, when about half of the ions remain and the increase in surface collisions has begun to clearly dominate that of grid transits.

We emphasize that these COMSOL simulations are collisionless and include only the vacuum electric field. This clear simplification was adopted as a first step in modeling our device and to maintain tractable computing times while considering a range of cathode and anode models. The modeling, therefore, does not incorporate effects related to space charge build-up, Debye shielding, or scattering and so can be used as an interpretive aid only with caution. We discuss some limitations related to neglecting space charge and Debye shielding in Sec. IV, while here noting that our simulations-despite these limitations-can nevertheless reproduce salient experimental results. With regard to treating the plasma as collisionless, the mean free path $\lambda$ of $\mathrm{D}_{2}$ neutrals and fast $\mathrm{D}$ ions is $\lambda \approx 45 \mathrm{~cm}$ and $\approx 60 \mathrm{~cm}$, respectively, at our typical operating pressure of $P \approx 2 \mu$ bar. This is comparable with the dimensions of the vacuum chamber, so collisions are not frequent. Given the predicted ion recirculation factor of $\sim 2.7$ (see Sec. III B), an average non-fusing ion born within half the anode radius will, in fact, collide with the cathode grid or voltage stalk before traversing a path length of $\lambda$.

\section{RESULTS}

\section{A. Discharge characteristics}

To establish the operational limitations of the experiment, we first note that the main adjustable parameters for any discharge are the neutral gas pressure $P$ and the supplied voltage $U$ and current $I$ for a given gas species and cathode grid. At pressures $P \lessgtr 50 \mu \mathrm{bar}$, the observed plasma breakdown voltage follows Paschen's law for plane-parallel electrodes but becomes largely independent of pressure at higher $P$ for any grid type, gas species, and anode configuration. This is similar to results for other IEC devices ${ }^{14}$ and suggests that the range in inter-electrode distances inherent in our setup does not qualitatively affect the breakdown characteristics compared to that observed in purely spherical geometries. Examples of the dependence of the maximum $U$ on $I$ at fixed pressure are shown in Fig. 3(a) for Ar discharges. These results imply an effective gas conductivity increasing with $U$ and $P$, with an exponentially declining value of $d U / d I=(4.9 \pm 0.9) \exp [(-0.51 \pm 0.05) P]$ in the units of Fig. 3. The observed linear relationships between $U$ and $I$ differ slightly from the $I \propto U^{3 / 2}$ behavior expected for an ideal space-charge limited ion flow between concentric spheres. ${ }^{15}$

The formation of ion microchannels through the cathode grid openings, the so-called "star mode" [Fig. 3(b)], occurs above a pressure-dependent voltage limit for all grids employed. In contrast, "jets" associated with a single grid opening in slightly anisotropic grids are mainly, though not exclusively, seen for the manually produced grids, attesting to the high symmetry of the $3 \mathrm{D}$ printed Ti grids. However, at certain pressures and low currents, discharges employing the smallest $(d=4 \mathrm{~cm})$ Ti grid can display up to four simultaneous "spray" jets that switch between nearly all grid openings on $\sim 0.1 \mathrm{~s}$ timescales [Fig. 3(c)]. This implies repeated formation of 1 to 4 current-carrying microchannels along constantly changing directions, with lifetimes that may be comparable to the typical collisional ionization timescale within the cathode. ${ }^{9}$ Similar phenomena, possibly related to variations in the energy distribution of electrons generated within the cathode, have recently been reported in other devices employing different cathode geometries. ${ }^{16}$ A possible correlation between jet shifts and variations in measured current will be explored in future work using rapid sampling of the current evolution.

Cathode grid heating due to ion bombardment may become significant during high-power operation, a common issue in gridded IEC devices. Visible heating is more prominent and occurs at lower supplied power for the smallest $d=4 \mathrm{~cm}$ Ti grid, presumably due to its 

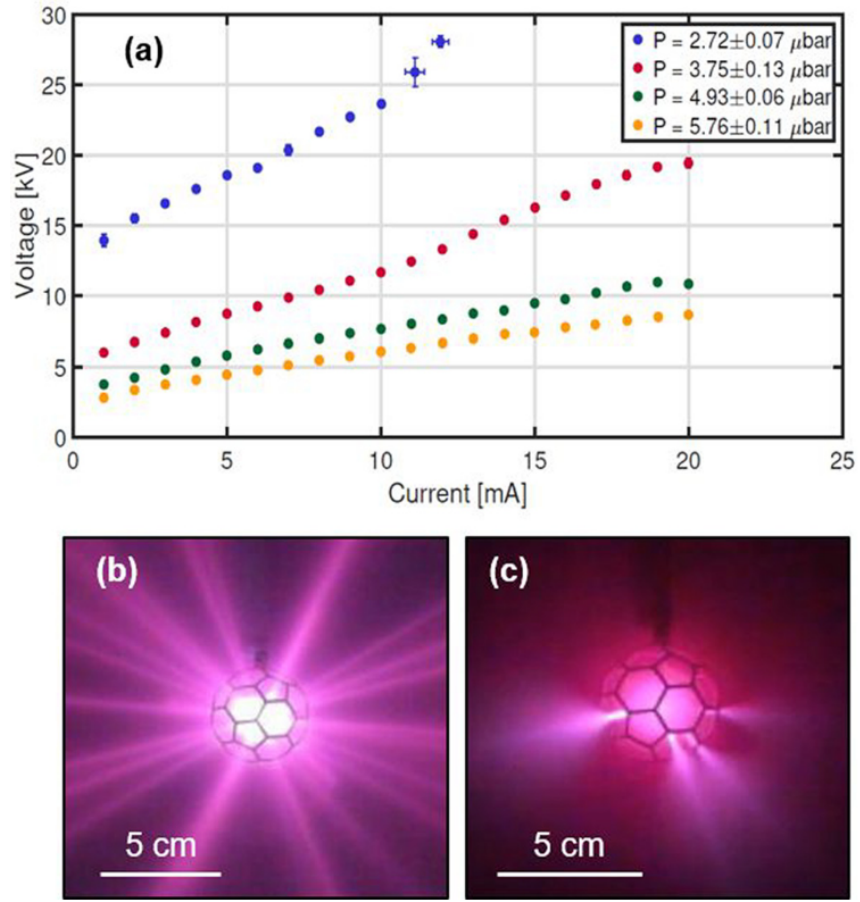

FIG. 3. U-I curves for Ar discharges with the $d=8 \mathrm{~cm}$ Ti grid at different pressures. Error bars representing the standard deviation of $I(t)$ and $U(t)$ across 1 min intervals are plotted but are mostly not visible. (b) Standard star-mode in a D discharge with the $d=8 \mathrm{~cm}$ Ti grid. (c) Multiple rapidly shifting spray jets in a $\mathrm{H}$ discharge with the $d=4 \mathrm{~cm}$ Ti grid $(P=2.6 \mu \mathrm{bar}, U=1 \mathrm{kV}$, and $I=5 \mathrm{~mA})$.

higher capacity for ion core convergence and its lower geometric transparency. Blackbody modeling of the associated $500-900 \mathrm{~nm}$ continuum emission measured by an OceanOptics optical-NIR spectrometer suggests grid temperatures reaching $T \approx 1350 \mathrm{~K}$ as shown in Fig. 4(a). This corresponds closely to the highest grid temperatures reported using pyrometer measurements in the Univ. of Wisconsin IEC device. ${ }^{17}$ After one year of operation with the tungsten grids, metal deposits had also developed on the vacuum windows, suspected to be caused by sputtering of the heated grids. As demonstrated in Fig. 4(b), this suspicion was confirmed by characterizing these deposits using an FEI Inspect scanning electron microscope equipped with an Oxford Instruments $\mathrm{x}$-max silicon drift detector. The results showed the deposits to consist of $>80 \% \mathrm{~W}$ by mass (with the remainder dominated by material in the window glass itself). For this reason, and to allow a safety margin to the $T \approx 1900 \mathrm{~K}$ melting point of the Ti grids, high-power operation with a visibly glowing cathode grid is usually confined to very brief periods. This is a main limitation on the maximum sustained neutron production rate of the current setup.

\section{B. Ion recirculation and core convergence}

An important factor for the overall fusion performance of IEC devices is the average number of transits through the cathode performed by the oscillating ions, i.e., the ion recirculation factor ${ }^{18}$ $\Gamma=\eta /\left(1-\eta^{2}\right)$. In the collisionless regime, this is determined by the vacuum electric field and hence by the transparency and symmetry of
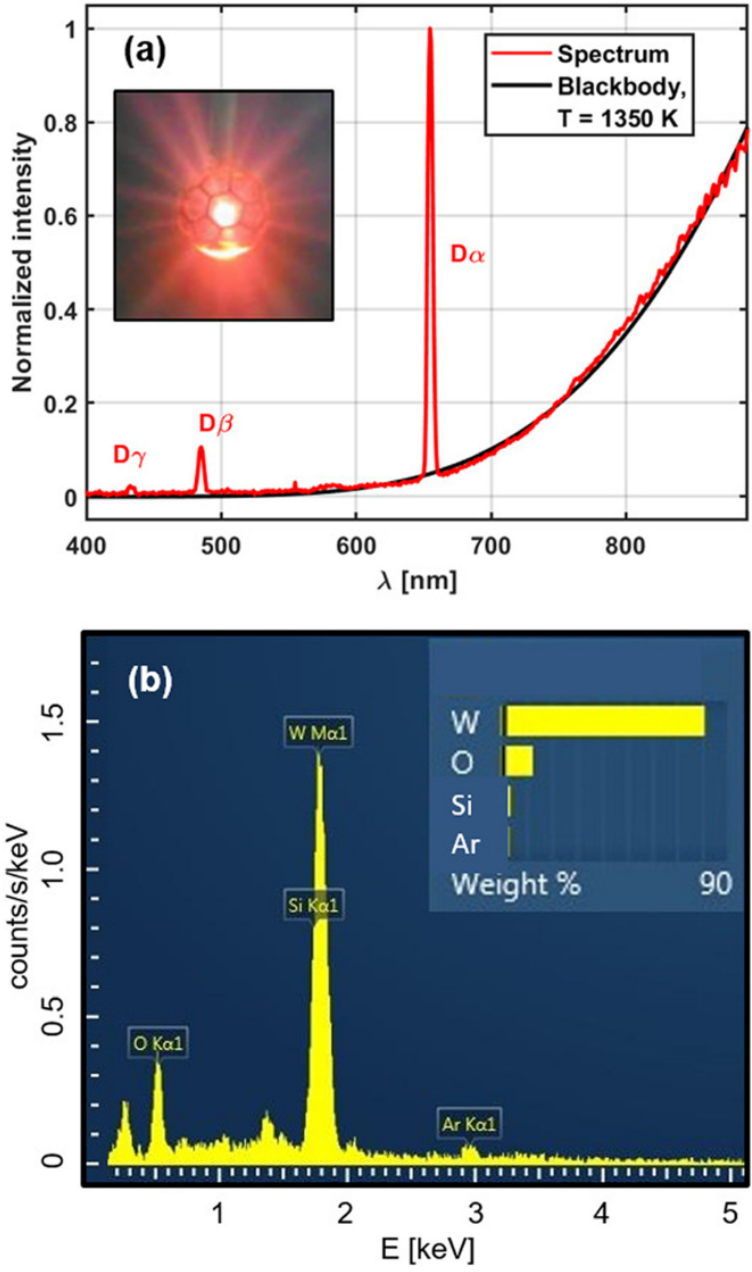

FIG. 4. (a) Normalized optical-NIR spectrum of a D plasma measured with a visibly glowing cathode grid (example photo of the $d=4 \mathrm{~cm}$ Ti grid shown as inset), across the wavelength range for which the spectrometer response is approximately uniform. The black curve shows a $T=1350 \mathrm{~K}$ blackbody spectrum scaled to match the measured continuum. (b) Results of scanning electron microscopy of a vacuum window covered by metal deposits after extended operation of the fusor. The composition of these deposits is heavily dominated by $\mathrm{W}$, with small amounts of $\mathrm{O}$ and Si from the borosilicate glass itself.

the cathode grid. Based on our COMSOL simulations, we evaluated the ion trajectories to estimate $\Gamma$ for our setup. Figure 5(a) shows the predicted static electric field in the full experimental geometry, for a simulation employing a model of the spherical $\mathrm{W}$ grid illustrated in Fig. 5(c). While viewports and inlets have no significant effect on the field geometry, the otherwise radial electric field generated by the cathode is seen to be distorted by the voltage stalk that supplies current to the cathode grid. This field distortion leads ions to collide with the stalk, a well-known issue for gridded IEC devices. ${ }^{2}$ By tracking the incident ion transits through the outer surface $S_{1}$ illustrated in Fig. 5(c), we derive $\Gamma=2.7 \pm 0.1$ for the spherical $\mathrm{W}$ grid, corresponding to an effective transparency of only $\eta \approx 0.83$ and an ion confinement time of $\sim 2 \mu \mathrm{s}$. In contrast, $\Gamma$ increases to $12.1 \pm 0.5$ in a 

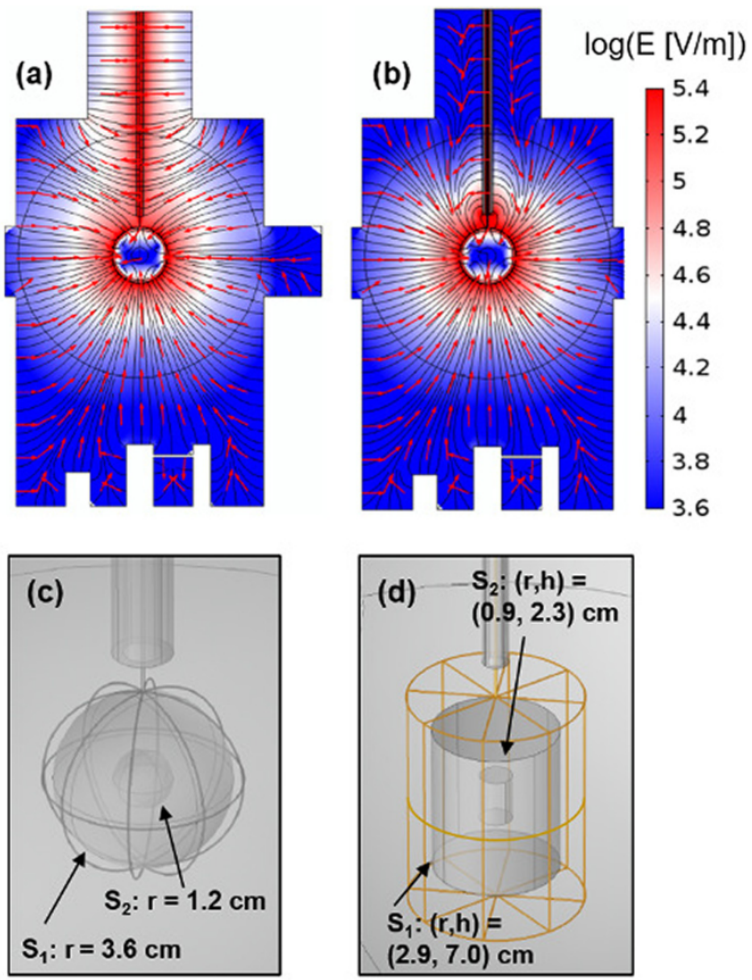

FIG. 5. (a) Simulated electric field in the fusor for an arbitrary cathode potential of $6 \mathrm{kV}$. Lines and arrows outline the local direction of the field. The black circle marks the extent of the uniform ion birth distribution assumed in some of the simulations (Sec. II B). (b) As (a), but if including a grounded metal shield within the dielectric surrounding the voltage stalk. (c) COMSOL model of the spherical W cathode grid and voltage stalk/quartz tube, along with the grid and core boundary surfaces $S_{1}$ and $S_{2}$ adopted for tracking ion passages. (d) As (c), but for the corresponding cylindrical grid. $S_{1}$ and $S_{2}$ are congruent with the relevant grid and hence match the geometry of the local electric field lines, while having similar surface areas and enclosed volumes between the two grids (to within $9 \%$ ).

simulation without the stalk, consistent with the value of $\Gamma=12.2$ anticipated from the geometric transparency $\eta=0.96$ of the modeled grid. Similar results are obtained for a model of the cylindrical $\mathrm{W}$ grid, shown in Fig. 5(d), as expected from its similar geometric $\eta$.

The stalk is, thus, a major limiting factor on ion recirculation and convergence, diverting ions toward it and reducing the ion flux through the grid in the upper half of the fusor. This also explains why visible grid heating due to ion impact is mainly localized at the bottom of the grids, regardless of the grid type and shape (see Fig. 4 for an example). As suggested in Fig. 5(b), it is possible to restore the radial symmetry of the electric field by surrounding the stalk with a grounded metal shield, here encased in a high-permittivity dielectric to prevent breakdown in the vacuum region between stalk/grid and shield. This also restores the predicted ion recirculation factor to a value of $\Gamma=12.6 \pm 0.5$, consistent with that inferred without a stalk, and reduces the fraction of lost ions associated with the stalk from $\sim 60 \%$ to $<1 \%$. Hence, this modification should assist in optimizing the performance of our device, which we will aim to test experimentally in future work.
Good ion core convergence may also facilitate high fusion rates in gridded IEC experiments. ${ }^{5}$ Angular momentum of the ions due to finite temperature, space charge effects, and inhomogeneities in the grid electric potential would reduce this convergence and lead to a cored ion density distribution. Quantifying this experimentally allows validation of our COMSOL simulations, while establishing the importance of collisional processes and identifying further points for performance optimization (see Sec. IV). The ion defocussing can be assessed based on the spatial distribution of visible light from excited neutrals. ${ }^{19}$ These neutrals are created by electron-impact excitation and from fast ions via charge exchange reactions such as $D^{+}+D_{2} \rightarrow D^{*}+D_{2}^{+}$, where $D^{*}$ represents an excited energetic neutral. ${ }^{20}$

Figure 6(a) shows example intensity profiles obtained from a $\mathrm{He}$ discharge in the star mode with the $d=8 \mathrm{~cm}$ Ti grid. The profiles were extracted horizontally along a direction not intercepting any visible ion microchannels. At fixed pressure, the profiles are seen to broaden with increasing $U$. This is illustrated in more detail in Fig. 6(b), which shows the derived half width at half maximum (HWHM) for profiles extracted across a range in $U$ and $P$. This width increases approximately linearly with voltage and additionally with pressure at fixed voltage, with both behaviors differing from results obtained elsewhere at significantly lower pressures. ${ }^{19}$ Outside the core, the measured intensity profiles $I(x)$ can be closely approximated by an exponential decline, $I(x) \propto \exp (\beta x)$, as shown in Fig. 6(c). In this region, the corresponding deprojected profile $I^{\prime}(r)$, obtained as the inverse Abel transform of $I(x)$,

$$
I^{\prime}(r) \propto-\beta \int_{r}^{\infty} \frac{\exp (\beta x)}{\sqrt{x^{2}-r^{2}}} \mathrm{dx} \propto-\beta K_{0}(\beta r),
$$

can, therefore, be described analytically using the modified Bessel function of the second kind of order $0, K_{0}$. However, the full profile must be deprojected numerically. The result, also shown in Fig. 6(c), displays an $\mathrm{HWHM} \approx 1.3 \mathrm{~cm}$, which provides an experimental estimate of the characteristic core radius $r_{c}$ in the ion density distribution.

This value can be compared to a simple theoretical estimate based on the assumption that conservation of ion angular momentum is the only contribution to ion defocussing in the core, yielding ${ }^{19}$

$$
r_{c} \approx \frac{3}{2} r_{b} \sqrt{\frac{k_{B} T_{i, \perp}\left(r_{b}\right)}{q \phi_{\mathrm{cat}}}},
$$

where $r_{b}$ is the ion birth radius (ideally equal to the anode radius), $k_{B}$ is Boltzmann's constant, $T_{i, \perp}\left(r_{b}\right)$ is the azimuthal ion temperature at $r_{b}, q$ is the elementary charge (assuming a singly ionized plasma), and $\phi_{\text {cat }}$ is the effective electric potential at the cathode. Note that $\phi_{\text {cat }}$ may be lower than the applied voltage by up to a factor of two, due to grid anisotropy and Debye shielding of the grid wires. ${ }^{19}$ If taking $\phi_{\text {cat }} \approx U / 2$ and considering singly ionized He with an assumed ${ }^{19}$ $T_{i, \perp}=0.1 \mathrm{eV}$, Eq. (2) would predict $r_{c} \lesssim 0.2 \mathrm{~cm}$ at $U \gtrsim 5 \mathrm{kV}$, i.e., significantly smaller than the measured estimate.

This discrepancy points to the additional defocussing effect of asymmetries in the cathode potential, unless ions in the DTU fusor are born with much larger azimuthal velocities than typically assumed for other similar devices. To elucidate this, we used our COMSOL simulations to estimate the average minimum distance $r_{\min }$ to the cathode center of ions that complete at least one core transit. From these collisionless simulations, in which ions are born at rest $\left(T_{i, \perp}=0\right)$, we find 

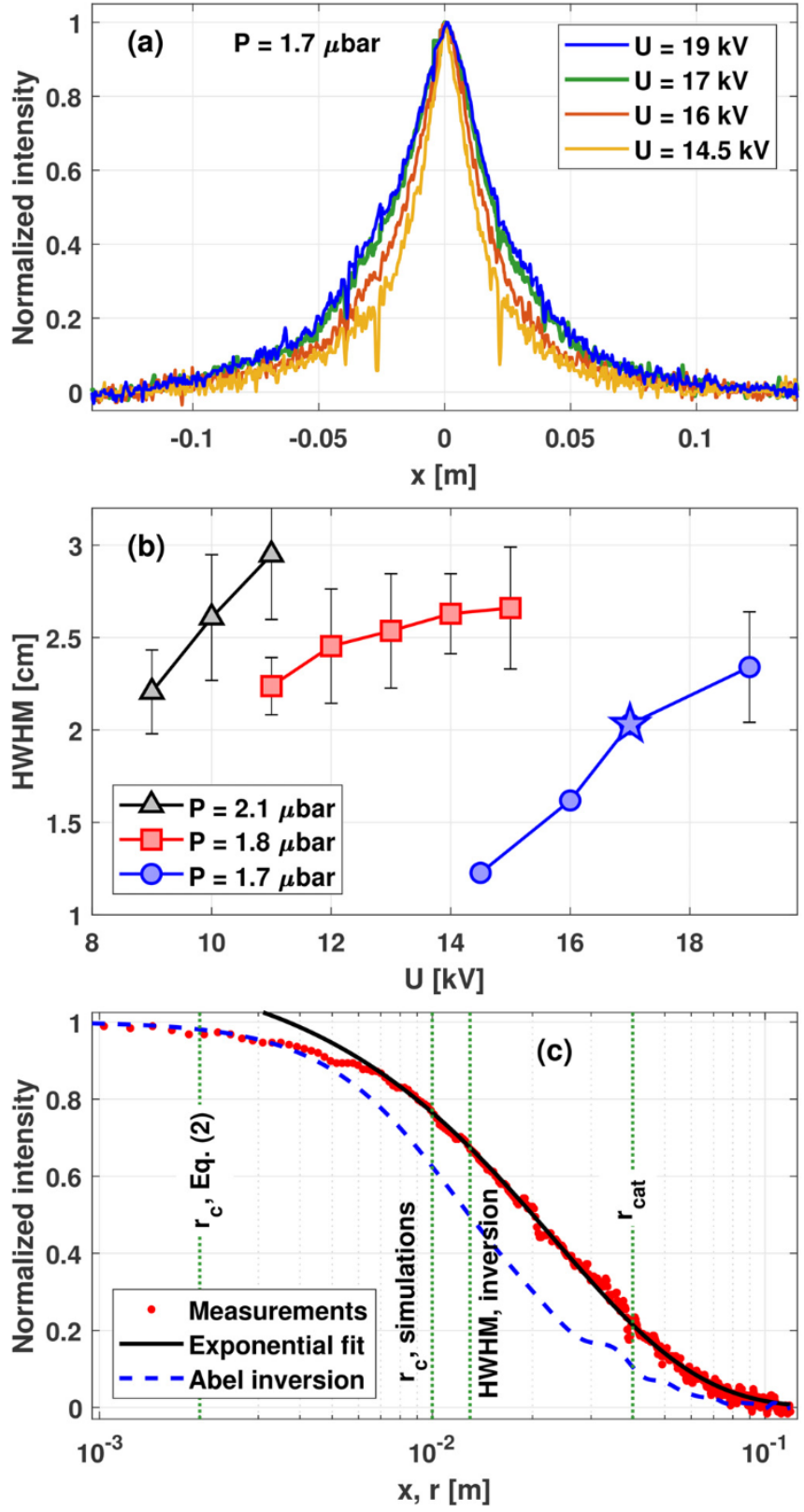

FIG. 6. (a) Normalized optical intensity as a function of projected horizontal distance $x$ from the cathode grid center for a He discharge at $P \approx 1.7 \mu$ bar. Local dips in the profiles are due to obscuration by grid wires. (b) Half-width at half maximum (HWHM) of intensity profiles as a function of $U$ for discharges with different $P$. Error bars indicate the error on the mean, derived from the rising and falling halves of each profile. (c) Normalized (projected) intensity distribution from the center of the cathode grid for the measurement at $P \approx 1.7 \mu$ bar and $U=17 \mathrm{kV}$ marked by a star in panel (b). The profile has been corrected for the presence of grid wires using linear interpolation. Also shown is an exponential fit to the data at $x>1 \mathrm{~cm}$, and the deprojected profile as a function of radial coordinate $r$. Vertical lines mark the cathode radius $r_{\text {cat }}=4 \mathrm{~cm}, H W H M=1.3 \mathrm{~cm}$ of the deprojected profile, and the estimates of $r_{c} \approx 1.0 \mathrm{~cm}$ and $r_{c} \lesssim 0.2 \mathrm{~cm}$ from our COMSOL simulations and Eq. (2), respectively. $r_{\min }=0.9-1.0 \mathrm{~cm}$ when employing a model of the spherical $\mathrm{W}$ grid listed in Table II and $r_{\min }=1.6-2.2 \mathrm{~cm}$ for the corresponding cylindrical grid. The range reflects the variation between the considered ion birth distributions, with the smaller values associated with the case of ions born at $r=1.5 r_{\text {cat }}$ (see Sec. II B). If identifying these results with $r_{o}$ the values for the spherical grid, thus, agree well with the experimental result, confirming the anisotropy of the electric potential as the main source of core defocussing. Indeed, the larger predicted value of $r_{c}$ for the cylindrical grid is to be expected, as the associated electric field is not completely radial in this case. This is corroborated by estimates of the time-averaged incident ion flux $j$ [ions s$~_{-1} \mathrm{~m}^{-2}$ ] through the boundary surfaces $S_{1}$ and $S_{2}$ shown in Figs. 5(c) and 5(d). Depending on the assumed ion birth distribution, we obtain ratios for the spherical grid of $j_{S 2} / j_{S 1}=4.1-5.5$, higher than those of the cylindrical grid, $j_{S 2} / j_{S 1}=3.3-3.5$.

\section{Neutron production rates}

The fusion reactivity is a direct measure of the performance of IEC devices, and its variation with experimental parameters provides crucial information for any optimization effort. The DTU fusor is currently capable of generating up to $10^{6}$ fusion neutrons $\mathrm{s}^{-1}$ in short intervals, with a typical maximum sustained rate of $\sim 2 \times 10^{5} \mathrm{~s}^{-1}$ as limited by grid heating. These neutrons may be produced in reactions between fast ions themselves, between fast ions and background neutrals, and between fast neutrals and background neutrals. In the first case, the fusion power would scale with measured cathode current as $P_{\text {fus }} \propto I^{2}$ at fixed core convergence, in the other cases as $P_{\text {fus }} \propto I$, if ignoring collisions and space charge effects. All other contributions, such as fast ion-fast neutral, fast neutral-fast neutral, or background-background, can likely be ignored, on account of low particle densities, low velocities, or low core convergence.

Neutron production rates (NPRs) have been measured for all cathode grids in the standard configuration (with the vacuum chamber acting as anode), as well as for a "nested" configuration with the $d=4 \mathrm{~cm}$ Ti cathode grid located inside the $d=16 \mathrm{~cm}$ Ti grid. In the nested configuration, the larger grid acts as an anode at ground potential. Despite the lack of 3D symmetry in the setup, no clear evidence of spatial anisotropy in the neutron distribution has been detected at equidistant locations with respect to the vacuum chamber. We, therefore, assume that neutrons are emitted isotropically, allowing straightforward conversion from measured to total rates. Most measurements presented here were conducted within a narrow pressure range of $P \approx 1.5-2.0 \mu$ bar, with some exceptions to be discussed.

Figure 7(a) shows the inferred NPR as a function of current $I$ within different voltage ranges for the $d=8 \mathrm{~cm}$ Ti grid. The measured rates scale approximately linearly with $I$ at comparable $U$ and $P$, and this applies to all grid configurations. This strongly suggests that fusion rates are dominated by reactions between fast particles (ions and charge-exchange neutrals) and background gas, as both theoretically expected and experimentally reported for most similar IEC devices. ${ }^{6,9}$ The results are also compared to predictions from a collisionless ion flow model, ${ }^{9}$ which assumes that the fusion reactivity is dominated by beam-background reactions taking place within the cathode, yielding

$$
N P R=\frac{I n_{0} r_{\mathrm{cat}} \sigma_{\mathrm{fus}}(E)}{2 q(1+\gamma)\left(1-\eta^{2}\right)},
$$



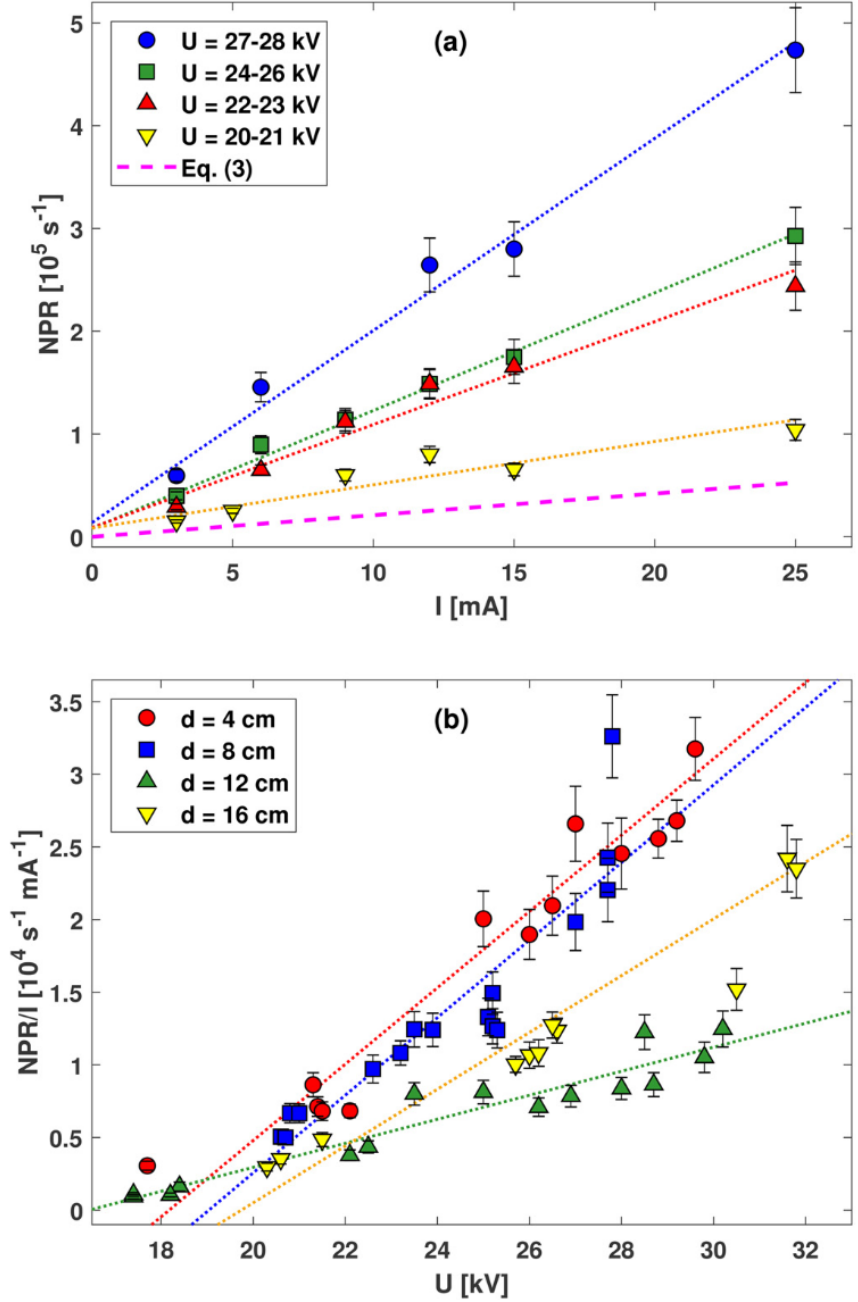

FIG. 7. (a) Neutron production rates as a function of current in different voltage ranges, measured with the $d=8 \mathrm{~cm}$ Ti cathode grid. (b) Scaled neutron production rates NPR/I vs voltage for the $3 \mathrm{D}$ printed Ti grids of varying size. Dashed lines represent linear fits to each measurement series, and error bars indicate $1 \sigma$ Poisson uncertainties on measured neutron rates.

where $n_{0}$ is the density of background neutrals, $\sigma_{\text {fus }}$ is the beambackground fusion cross section, ${ }^{21}$ here conservatively computed at the maximum beam energy $E=q U$ and median voltage $U \approx 24 \mathrm{kV}$, $\gamma \approx 1$ is the secondary electron emission coefficient of $\mathrm{Ti}^{22,23}$ and where we have used the geometric value of $\eta=0.93$ (Table II). The observed fusion rates generally exceed this expectation by a factor of $\sim 5$ at comparable $U$, thus corroborating the well-established result that fusion in IEC devices is more efficient than expected from simple theoretical arguments. ${ }^{9}$

Given the linear scaling of the NPR with $I$, the performance of all grids can be compared by considering the associated scaled reactivity, $N P R / I$. Figure 7(b) illustrates this quantity for the different 3D printed cathode grids. Measured fusion rates are seen to scale approximately linearly with $U$ across the limited voltage range considered here. Despite the scatter, it is clear that the smaller $d=4$ and $8 \mathrm{~cm}$ grids perform better than the two larger grids, although there is no monotonic relation between grid diameter and NPR/I at fixed $U$. Hence, our results suggest an optimal ratio $r_{\text {cat }} / r_{\text {an }} \leqq 0.25$ for our device, similar to findings in other experiments. ${ }^{9}$ This differs from the $N P R \propto d^{-0.4}$ scaling reported elsewhere, ${ }^{24}$ and from the results of several other experiments $\mathrm{s}^{2,7,8}$ that show higher neutron rates as $r_{\text {cat }} / r_{\text {an }}$ approaches $\approx 0.5$. This might be explained by differences in ion birth profiles and in the frequency of collisional processes and is testament to the significant variation in discharge characteristics with the specific setup of IEC devices.

The cathode and anode geometry, as determined by the shape of the electrodes and the number of grid openings, can also affect fusion reactivity. Previous results in this regard also seem highly devicedependent. Some studies ${ }^{9,19}$ concluded that the number of cathode grid openings does not clearly affect the NPR, whereas others observed a clear tendency toward a higher NPR for more symmetric grids. ${ }^{25}$ Measurements for spherical vs cylindrical cathode grids of comparable base radii have also returned conflicting results. ${ }^{6,8}$ Finally, experiments using a fixed cathode radius ${ }^{4}$ found no evidence of variations in neutron production for a range of anode radii, whereas others ${ }^{8}$ reported a higher neutron rate when modifying anode and cathode radii from a radius ratio of $r_{\text {cat }} / r_{\text {an }}=0.2$ to 0.5 .

To explore this issue for the DTU device, Fig. 8 presents the fusion performance with respect to both cathode and anode geometry. Comparing the $d=8 \mathrm{~cm} 3 \mathrm{D}$-printed Ti grid (32 openings) to the similarly sized W grid (16 openings) in Fig. 8(a) shows that the smaller and more symmetric cathode wire spacing of the Ti grid on average results in a $25 \%$ higher NPR. Hence, our use of these soccerball-like grids presents a distinct improvement with respect to our manually manufactured grids. An even more pronounced difference, however, is seen in Fig. 8(b) between the otherwise identical spherical and cylindrical W grids. The former yields a systematically higher NPR, qualitatively similar to other findings. ${ }^{8}$ The two measurement series in Fig. 8(b) were performed at higher pressures though, $P \approx 7-9 \mu \mathrm{bar}$, than the remaining results presented here, and this may be relevant for their interpretation as discussed in Sec. IV. In addition, several data points for the cylindrical grid were obtained at pressures differing by $0.5-1.0 \mu$ bar from the spherical-grid data at similar $U$, and these are flagged as empty circles in Fig. 8(b). If considering all data within the $U=23-29 \mathrm{keV}$ range, the NPR for the spherical grid is on average higher by a factor of $\sim 2.4$ (dashed line in the figure), potentially increasing to $\sim 3.5$ at $U \gtrsim 30 \mathrm{keV}$. It remains higher by a factor of $\sim 1.5$ if considering only the similar- $P$ measurements within $U=23-26 \mathrm{keV}$ (dash-dotted line).

With regard to the anode, Fig. 8(c) compares the case of using the cylindrical vacuum chamber $(d \approx 36 \mathrm{~cm})$ as the anode to that of the two-grid "nested" configuration with the $d=16 \mathrm{~cm}$ Ti grid acting as the anode. A significantly higher NPR is seen in the former case by $\sim 60 \%$ on average, suggesting that the use of concentric spherical electrodes does not improve fusion performance in our setup. This is plausibly a result of lower ionization rates in the background gas between the electrodes in the two-grid configuration, for which the electrode distance is only $6 \mathrm{~cm}$. This is well below the corresponding minimum values of $10-16 \mathrm{~cm}$ for any cathode grid with the vacuum chamber as the anode, and far below the mean free path of $\lambda \sim 0.5 \mathrm{~m}$ at $P \approx 2 \mu \mathrm{bar}$ (Sec. II B). Obtaining and sustaining plasma breakdown at $P \approx 2 \mu \mathrm{bar}$ based on impact ionization should, thus, be more difficult in the twogrid configuration, and this was indeed observed experimentally. 

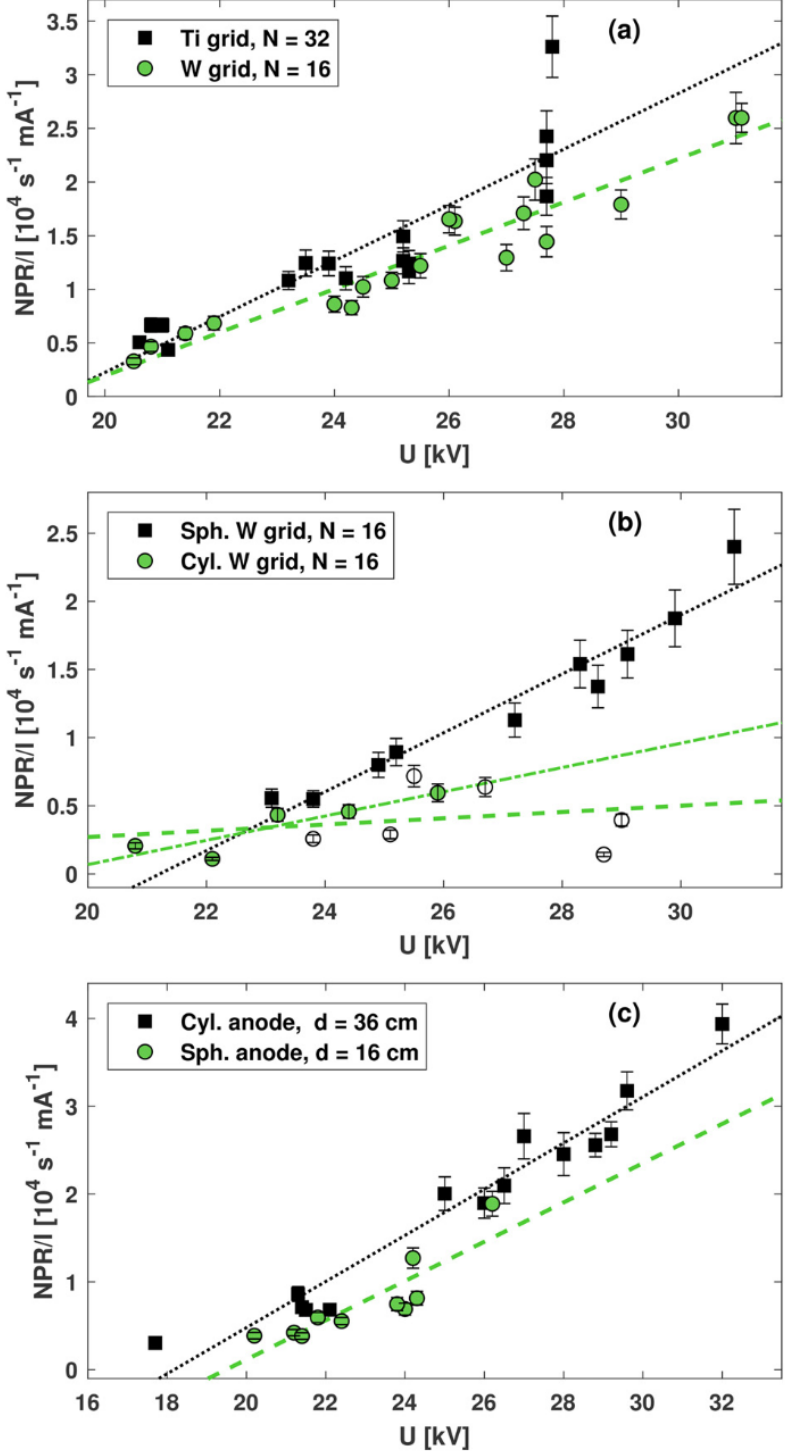

FIG. 8. Dependence of scaled neutron production rates on cathode and anode geometry. (a) NPR/I for the 3D printed $d=8 \mathrm{~cm}$ Ti cathode grid (32 openings) and the corresponding manually produced $\mathrm{W}$ grid (16 openings) as functions of voltage. Lines show the respective best-fit linear relations, and error bars represent $1 \sigma$ Poisson uncertainties on measured neutron rates. (b) As (a), but for the spherical and cylindrical W grids. For the cylindrical grid, the dashed line here shows the linear fit to all data (filled and empty circles), while dash-dotted line represents a fit to those measurements conducted at pressures identical to those underlying the spherical-grid results (filled circles only); see the text for details. (c) As (a), but comparing results for the $d=4 \mathrm{~cm}$ Ti cathode grid, using as the anode either the $d \approx 36 \mathrm{~cm}$ cylindrical vacuum chamber or the corresponding spherical $d=16 \mathrm{~cm}$ grid.

\section{Electron dynamics}

Internal (unassisted) IEC discharges rely in part on free electrons to generate and maintain the plasma. Large electron currents $I_{e}$ are nevertheless undesirable, as they reduce the ion current $I_{i}$ available for fusion at fixed measured cathode current $I=I_{i}-I_{e}$. Basic insight into the sources and dynamics of free electrons in our device can be gained from the measured $\mathrm{x}$-ray spectra, produced by bremsstrahlung losses as electrons collide with the vacuum vessel wall. Figure 9(a) shows spectra obtained for a D plasma, corrected for background, detector response, and window transmission $\tau_{w}$ (which increases from $\sim 5 \%$ to $75 \%$ across the plotted energy range, as shown in the inset). Furthermore, Fig. 9(b) shows the maximum photon energies robustly detected at $5 \sigma$ above the background as a function of voltage. The spectra broadly resemble anode bremsstrahlung spectra, ${ }^{26}$ with a linear decline at high energies, a high-energy cutoff that corresponds closely to the maximum energy $E_{\max }=q U$ supplied to individual electrons by the voltage drop across the electrodes, and with a photon energy $E_{\text {peak }}$ at peak intensity that depends only weakly on $U$. The absence of a significant signal at photon energies well above $E=q U$ suggests that any bremsstrahlung contribution from fusion products impinging on the anode wall is small.

While the result in Fig. 9(b) does not itself constrain the electron birth distribution, it does provide experimental evidence for electron release at the cathode and acceleration of at least some electrons through the full electric potential. We have observed no spatial
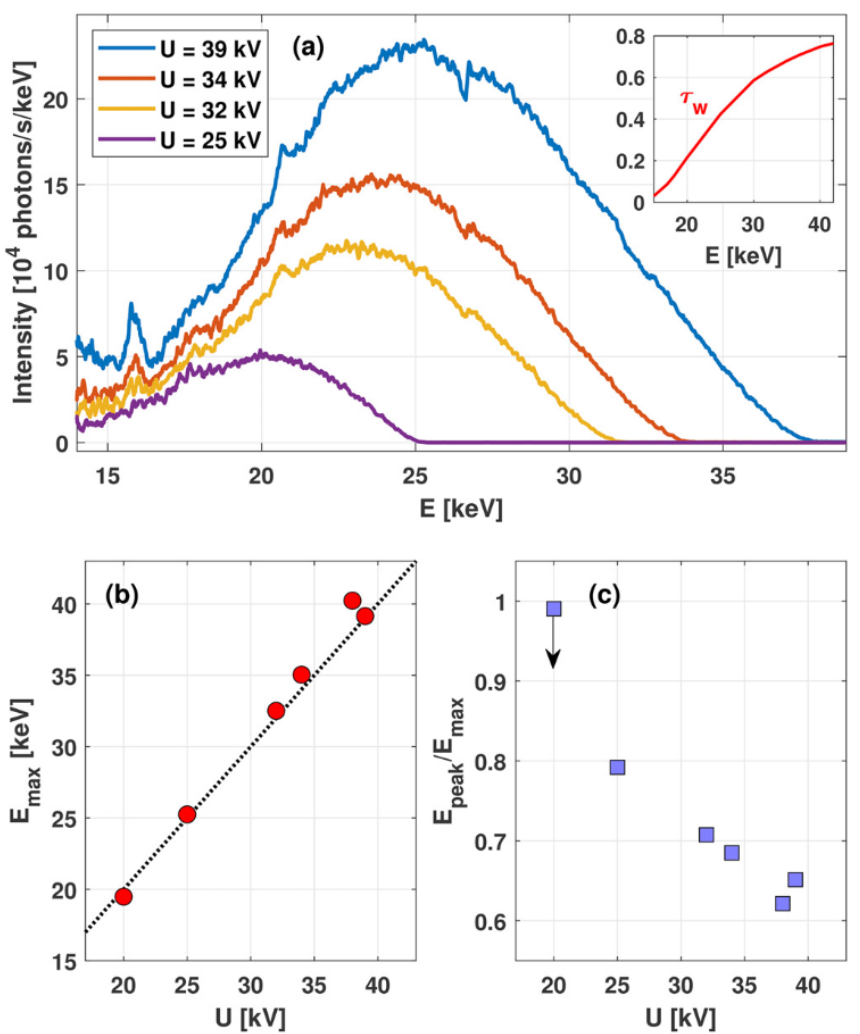

FIG. 9. (a) Example $\mathrm{x}$-ray spectra from a $\mathrm{D}$ plasma at $I=15 \mathrm{~mA}, P \approx 1 \mu$ bar. The small dip at $E \approx 26 \mathrm{keV}$ is an artifact caused by a slightly insufficient correction of fluorescence emission in the CdTe detector. The inset shows the transmission curve $\tau_{w}(E)$ of the vacuum window. (b) Maximum detected photon energy $E_{\max }$ for all spectra as a function of voltage $U$. The dotted line shows the relation $E_{\max }=q U$. (c) Photon energy at peak intensity $E_{\text {peak }}$ relative to $E_{\max }$, for all spectra with $E_{\text {peak }} \gtrsim 20 \mathrm{keV}$ corresponding to $\tau_{w} \gtrsim 0.2$. The result at $U=20 \mathrm{kV}$ is an upper limit due to the associated low value of $\tau_{w}$. 
anisotropy in the measured intensity of $\mathrm{x}$ rays penetrating the different vacuum windows for discharges using the spherical $\mathrm{W}$ grid. Hence, the total $\mathrm{x}$-ray power emitted at $E>15 \mathrm{keV}$ (where window transmission is non-negligible) can be estimated from the spectra in Fig. 9(a) assuming isotropic emission. This, in turn, yields a lower limit to the electron current $I_{e}$ in the experiment of $I_{e}>(0.1-0.6) \times 10^{-6} \mathrm{~mA}$ for the voltage range shown in Fig. 9(a). For comparison, an upper limit to the thermionic emission current due to thermal release of electrons from the heated cathode is ${ }^{27}$

$$
I_{e, t h}=A_{\text {grid }} \frac{4 \pi m_{e} q k_{B}^{2}}{h^{3}} T^{2} \exp \left(-\frac{W_{\text {cat }}}{k_{B} T}\right),
$$

where $A_{\text {grid }}$ is the grid surface area, $m_{e}$ is the electron mass, $h$ is Planck's constant, $T$ is the grid temperature, and $W_{\text {cat }}$ is the work function of the cathode material $\left(W_{\text {cat }}=4.3 \mathrm{eV}\right.$ for $\mathrm{Ti}$ and $4.8 \mathrm{eV}$ for $\mathrm{W})$. For the spherical $\mathrm{W}$ grid $\left(A_{\text {grid }}=27.6 \mathrm{~cm}^{2}\right)$ and an extreme case with $T \approx 1350 \mathrm{~K}$ (cf. Fig. 4 ), we obtain $I_{e, t h} \lesssim 8 \times 10^{-6} \mathrm{~mA}$. In this case, we cannot formally exclude the possibility that the entire electron current is driven by thermionic emission, but the current is far below the total measured current and should not in itself affect the ion current and the associated fusion performance adversely. Under normal operation, we can expect $T \lesssim 1000 \mathrm{~K}$, implying $I_{e, t h}<1 \mathrm{nA}$ for all grids. In this case, $I_{e}$ must be dominated by electrons produced by impact ionization of the background gas and through secondary electron emission from ion and photon impact on the grid.

$\mathrm{X}$-ray lines from recombination and subsequent de-excitation of $\mathrm{Fe}$ atoms in the steel $(>98 \% \mathrm{Fe})$ walls occur at lower energies than shown here, for which the transmission of the vacuum windows becomes negligible. In principle, the shape of the measured $\mathrm{x}$-ray spectra nevertheless still contains information on the electron velocity distribution function $f_{e}$ at the anode. Specifically, the spectra are seen to broaden relative to their peak energy with increasing $U$ across the observed voltage range [Fig. 9(c)], which might reflect changes in the shape of $f_{e}$ with $U$. However, extracting this information would require forward modeling involving Monte Carlo simulations of the electron penetration and photon reabsorption in the anode material, and this is left for future work.

\section{DISCUSSION}

The observed linear dependence of measured fusion rates on cathode current indicates that fast particle-background reactions dominate the fusion reactivity, as in virtually all other gridded IEC devices. A further argument in favor of this comes from the collisionless ion flow model underlying Eq. (2). For a grid with $\eta \approx 0.95$ and $\phi_{\text {cat }} \approx U / 2$ embedded in a singly ionized He plasma with $I=15 \mathrm{~mA}$, $U=15 \mathrm{kV}$, and a measured core radius of $r_{c} \approx 1.3 \mathrm{~cm}$ (Sec. III B), this model would predict a core ion density ${ }^{19}$ of

$$
n_{\mathrm{ic}}=\frac{I \eta}{\pi r_{c}^{2} q^{3 / 2}\left(1-\eta^{2}\right)(1+\gamma)} \sqrt{\frac{m_{i}}{2 \phi_{\mathrm{cat}}}} \approx 1 \times 10^{15} \mathrm{~m}^{-3},
$$

where $m_{i}$ is the ion mass and other quantities have been defined above. At the considered operating pressures of $P \approx 2 \mu \mathrm{bar}$, this corresponds to a core ionization fraction $n_{\mathrm{ic}} / n_{0} \approx 3 \times 10^{-5}$, with currents of $I>200 \mathrm{~A}$ required to achieve $n_{\mathrm{ic}}>n_{0}$.

Other than increasing the ion current (e.g., through external ion injection), promoting beam-beam fusion in our device and gaining access to the more efficient $P_{\text {fus }} \propto I^{2}$ regime would, thus, require two further modifications at fixed voltage and grid diameter: eliminating the detrimental impact of the voltage stalk on the electric potential (increasing the effective $\eta$ ) and reducing grid wire spacings and hence $r_{c}$ without compromising grid transparency (i.e., using thinner wires) or structural integrity. While 3D-printed cathode grids enable a clear enhancement in fusion performance in our case, further development of this manufacturing technique would, thus, be required to suppress ion defocussing by several orders of magnitude.

In addition to the impact of wire spacing, our measurements of neutron production rates show a dependence on both size and shape of the applied cathode grids. Relatively small spherical grids clearly outperform other configurations, presumably due to their enhanced capacity for increasing $\Gamma$ and decreasing $r_{c}$. However, another highly significant difference is seen between the spherical and cylindrical cathode grids of similar base radius, with the former enabling neutron rates that are up to $\sim 3.5$ times higher at fixed voltage. In an attempt to understand this and explore possible implications, we again turn to our COMSOL simulations. Despite their limitations, discussed in more detail below, these simulations produce good quantitative agreement with the measured ion core radius and can explain the asymmetry of the observed grid heating (Sec. III B), thus lending credibility to this approach.

The simulated ion positions and velocities can be used to estimate the spatial distribution of beam-background fusion rates per unit volume,

$$
R_{\text {fus }}(r)=n_{0} n_{i}\langle\sigma v\rangle=n_{0} \int \sigma_{\text {fus }}(v) v(r) f(v, r) d v,
$$

for ions with density $n_{i}(r)$ and distribution function $f(v, r)$ reacting with a uniform background gas of density $n_{0}$ with negligible velocities. We also compute the volume-integrated values,

$$
R_{\mathrm{V}}\left(r^{\prime}<r\right)=4 \pi \int_{0}^{r} R_{\text {fus }}\left(r^{\prime}\right) r^{\prime 2} d r^{\prime}
$$

with both quantities averaged across the $t=1.5 \mu$ s simulation time. The results are shown in Fig. 10(a) for the modeled spherical and cylindrical $\mathrm{W}$ grids. Here, we have scaled all ion velocities to a grid potential of $U=25 \mathrm{kV}$, representative of the measurements in Fig. 8(b). The predicted $R_{\text {fus }}(r)$ peaks within the cathode for either grid and declines rapidly with the radius, in agreement with spatially resolved fusion product measurements at other IEC devices. ${ }^{28}$ The lower $R_{\text {fus }}$ for the case with a localized ion birth profile is mainly caused by lower ion velocities, as no ions are accelerated through the full potential in this case.

Despite the small volume enclosed by the cathodes, Fig. 10(b) suggests that $70 \%-90 \%$ of all fusion reactions take place within the cathode region for either ion birth distribution. Figure 10(c) shows the ratio of the total enclosed fusion rates $R_{\mathrm{V}}(<r)$ between the spherical and cylindrical grids, indicating values $R_{\mathrm{V}, \text { sph }}(<r) / R_{\mathrm{V}, \text { cyl }}(<r) \gtrsim 2$ within the cathodes. This ratio declines to $\approx 1$ well outside the cathodes, a variation that is primarily due to differences in core ion density between the grids (cf. Sec. III B), as the mean ion velocities induced by the two grids are comparable throughout this radial range. Hence, from these simulations, we should expect comparable total fusion rates with the two grids. 

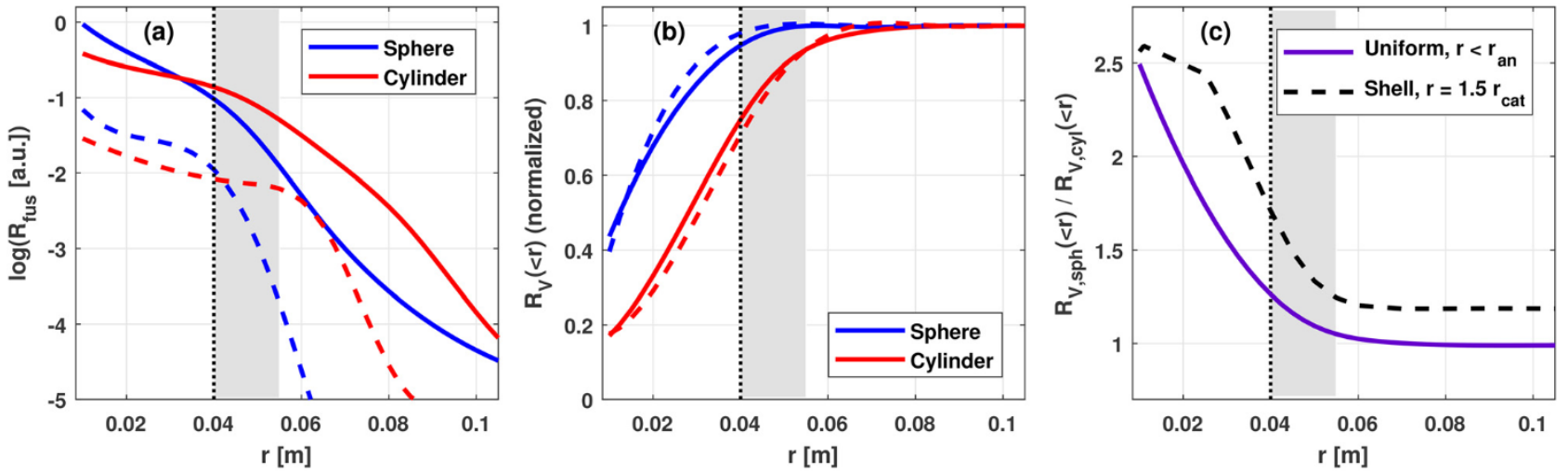

FIG. 10. Fusion reaction rates implied by our COMSOL simulations, assuming beam-background fusion and an ion birth profile that is either flat (solid lines) or localized at $r=1.5 r_{\text {cat }}$ (dashed lines). (a) Radial $R_{\text {fus }}(r)$ for the spherical and cylindrical cathode grids, all normalized to the central $R_{\text {fus }}$ of the spherical grid for a uniform ion birth distribution. (b) $R_{\mathrm{V}}$, the volume-integrated $R_{\text {fus }}$ within $r$ (normalized), for both grids. (c) Ratio of $R_{\mathrm{V}}$ for the spherical vs cylindrical grids. In all plots, $r_{\text {cat }}=0.04 \mathrm{~m}$ for the spherical grid is marked by a dotted line, and the corresponding range in $r_{\text {cat }}=0.04-0.055 \mathrm{~m}$ for the cylindrical grid by the gray shaded region. The small non-zero fusion rates outside $r=1.5 r_{\text {cat }}$ for the simulations with a localized ion birth distribution are associated with ions attracted toward the voltage stalk.

This contrasts with the experimental results, which imply average total ratios $R_{\mathrm{V}, \mathrm{sph}} / R_{\mathrm{V}, \mathrm{cyl}} \gtrsim 1.5$, potentially extending up to $\approx 3.5$ at $U \sim 30 \mathrm{keV}$. The two estimates can be reconciled if the simulations overpredict either $R_{\text {fus,cyl }}$ throughout or $R_{\text {fus }}$ for both grids outside a fixed radius of $r \approx 3-4 \mathrm{~cm}$. The latter case would imply a strong core localization of the total DD fusion rate, with fusion reactivity heavily dominated by the region within $r_{\text {cat }}$. This could trivially be the case if ionization takes place preferentially at and immediately outside the cathode or if the geometric arrangement of our neutron detector is preferentially sensitive to neutrons from the core region. Direct measurements of the ion birth profile or MCNP simulations accounting for the directional dependence of neutron thermalization in the detector moderator could test these possibilities.

An alternative explanation is that collisional processes such as charge exchange preferentially reduce the typical ion velocities and fusion reactivities outside the cathode compared to our collisionless predictions. The plasma is not entirely collisionless with respect to neutral particle interactions, as mentioned in Sec. II B and as evidenced by the increase in the measured ion core radius $r_{c}$ with pressure at fixed voltage, along with the departure from the scaling with $U$ expected from the collisionless model underlying Eq. (2). This could be particularly relevant for the measurements in Fig. 8(b), which were conducted at higher pressures than the remaining experiments. While the direction of motion of fast neutrals created via charge-exchange or dissociative collisions is similar to those of the incident fast ions, ${ }^{29}$ the net result of such collisions is a loss of ion energy and confinement. We evaluated the spatial dependence of this at constant neutral density, using the simulated ion velocities coupled with the cross section $\sigma_{c x}(v)$ for change exchange of atomic D on D. ${ }^{24}$ This reaction has the highest cross section among the ones involving the different $\mathrm{D}$ species $\left(D, D_{2}, D_{3}\right)$ at the relevant ion energies. The results suggest that the charge exchange probability $P \propto n_{0}\left\langle\sigma_{c x} v\right\rangle$ peaks within $r_{\text {cat }}$ but is only moderately dependent on radial location for either grid (to within $\sim 20 \%$ ) and is only slightly higher for the cylindrical than the spherical grid (to within $\sim 10 \%$ ).

It is, thus, not clear that charge exchange processes alone could modify the modeled fusion reactivity to match our measurements for the spherical vs cylindrical grid. However, more detailed multi-species modeling including ion-neutral collisions would be necessary to test this, coupled with more extensive NPR measurements with both grids across a broader pressure range. As a more direct measure, spatially resolved measurements of fusion protons could help test our findings. ${ }^{28,30}$ For now, we note that our tentative evidence for strongly core-localized fusion reactivity seems to agree with certain other measurements $^{30}$ and model results ${ }^{31}$ and would point to fast ion-neutral rather than fast neutral-neutral as the dominant fusion reaction. However, such core localization would differ significantly from-less model-dependent-results for other devices. ${ }^{9}$ While it may also be at odds with recent results suggesting a significant fusion contribution related to D atoms adsorbed/embedded in the inner surface of the anode, ${ }^{32}$ we reiterate that our $\mathrm{x}$-ray measurements show no evidence for such a contribution in our experiment (Sec. III D).

The possibility that the plasma significantly alters the electric potential compared to our vacuum-field calculations could also play a role. As mentioned, our COMSOL simulations represent noninteracting test particles orbiting in the vacuum electric field and so do not incorporate effects of space charge or Debye shielding. For an assumed ion density and electron temperature immediately outside the cathode grid of $10^{14}-10^{15} \mathrm{~m}^{-3}$ and $10 \mathrm{eV}$, respectively, the electron Debye length is $\lambda_{D} \approx 0.1-0.2 \mathrm{~cm}$. This is larger than the typical interion distance of $\lambda_{\mathrm{ii}} \sim 10^{-5} \mathrm{~m}$, but smaller than the linear dimensions of the grid openings of $1-3 \mathrm{~cm}$. Due to the $\lambda_{D} \propto\left(T_{e} / n_{e}\right)^{1 / 2}$ dependence, $\lambda_{D}$ is expected to increase with radius such that $\lambda_{D}>\lambda_{\mathrm{ii}}$ everywhere outside the cathode. As a consequence, charged-particle interactions and Debye shielding of the grid wires cannot formally be neglected in our experiment and should be included in a more selfconsistent fluid or particle-in-cell model of the fusor plasma.

For the recirculating ions, Debye shielding would reduce the effective potential between the grid wires and hence the average ion speed. The shielding may also reduce the frequency of ion collisions with the grid and voltage stalk, thereby boosting the recirculation factor $\Gamma$ compared to our estimates. The fact that our COMSOL simulations can nevertheless reproduce the measured ion core radii and the observed localized grid heating due to ion bombardment (Sec. III B) 
suggests that Debye shielding does not substantially affect the global dynamics of recirculating ions in our device.

Inside the cathode grid, space charge build-up of ions could result in the formation of a virtual anode, ${ }^{19}$ another mechanism not captured by our simulations. In fact, recent experimental work suggests that a significant component of the ion flow may originate within the center of the cathode and accelerate outward due to the formation of a virtual anode. $^{33}$ This would be distinct from the convergent ion flow picture resulting from our COMSOL simulations and could be tested experimentally by potential profile measurements inside the cathode. However, in this divergent ion flow scenario, we would expect any visible grid heating to occur uniformly across the grid, contrary to our observations that are consistent with the convergent ion flow predicted by COMSOL. Furthermore, it is unclear if the presence of a significant virtual anode and a predominantly divergent ion flow in our device would produce the good agreement between observed and simulated ion core radii, the linear dependence of neutron rates on current, or the difference in these rates produced by our spherical and cylindrical grids.

A final point concerns the contribution of surface fusion involving atoms adsorbed on or embedded in the cathode grid, which could contribute to the core localization of fusion rates indicated by our results. A recent study suggests that up to $75 \%$ of the fusion reactions could be accounted for by surface fusion under certain conditions, ${ }^{10}$ but also that cathode heating reduces these rates due to thermal desorption of $\mathrm{D}$. The fact that our highest neutron rates are seen for the Ti grids, which both have the smallest grid surface areas and are most prone to visible heating at fixed input power, suggests that surface fusion involving adsorbed/embedded D on the cathodes is not a major contributor to the fusion reactivity in our experiment. This is corroborated by the higher neutron rates seen for the spherical $\mathrm{W}$ grid $\left(A_{\text {grid }} \approx 28 \mathrm{~cm}^{2}\right)$ relative to the cylindrical one $\left(40 \mathrm{~cm}^{2}\right)$.

\section{SUMMARY AND CONCLUSIONS}

The DTU cylindrical fusor, operated since 2017, joins a relatively small community of university-run experiments on electrostatic inertial confinement of fusion plasmas. Distinct features of our experiment include the novel use of 3D-printed soccerball-like cathode grids, which yield fusion neutron rates that are $25 \%$ higher than those achieved using a similar-sized manually manufactured grid with larger grid openings. Further increases in fusion rates may be expected if this grid manufacturing technique can be developed to produce grids with more openings at similar or higher geometric transparency.

Despite the use of these grids and the cylindrical anode geometry, conditions for plasma breakdown and retention are qualitatively similar to those reported in other IEC devices at similar pressures. Cathode grid heating due to ion bombardment is the main limitation on highpower operation and sustained neutron production in the device. Indeed, electron microscopy provides direct evidence of heavy-ion sputtering off the cathode grids, and x-ray measurements show that impact-induced secondary electron emission from the grid dominates over thermionic emission under normal operating conditions.

In addition to the impact of grid wire spacing, the observed neutron production rates also depend on the size and shape of the cathode and anode, with smaller cathode grids $\left(r_{\text {cat }} / r_{\text {an }} \lesssim 0.25\right)$ yielding higher rates than do relatively large grids, as opposed to the case for a number of spherical IEC devices. ${ }^{2}$ In contrast, replacing the cylindrical anode wall with a spherical gridded anode lowers the observed neutron rates by a factor of $\sim 1.6$.

The linear scaling of measured neutron rates with current and the low estimated ionization fraction of $<10^{-4}$ identify the main fusion reactants as fast particles (ions and charge-exchange neutrals) fusing with background neutrals. A significant difference in neutron rates is observed between spherical and cylindrical cathode grids (the latter congruent with the anode) of similar base radius, with the former clearly outperforming the latter. This contrasts with the results reported for other cylindrical IEC devices, ${ }^{6}$ but may be explained if the total fusion reactivity is strongly core-localized. If so, fusion reactions are likely dominated by ion-neutral rather than fast neutral-neutral interactions. Future spatially resolved measurements of the distribution of fusion products in the device will help to test this. This must be coupled with more detailed modeling of ion collisions and electron bremsstrahlung losses in order to obtain a more complete picture of particle confinement and fusion reactivity in our fusor.

COMSOL simulations of ion orbits in the vacuum electric field in the fusor predict a characteristic core radius of the convergent ion flow in good agreement with measurements based on deprojecting the distribution of visible light. This confirms asymmetries in the grid electric potential as a main obstacle toward efficient ion convergence and generation of beam-beam reactions in gridded IEC devices. The simulations also suggest that the voltage stalk associated with the cathode grid acts as a major ion sink. Ion collisions induced by this stalk reduce the average number of ion core transits by a factor of $\sim 4$, the effective grid transparency from $\sim 95 \%$ to $\sim 83 \%$, and cause ions to collide with the grid preferentially from directions opposite to the stalk, as observed. This suggests that simple reevaluation of the stalk design, such as including a grounded metal shield, could significantly improve ion recirculation in IEC devices such as ours.

\section{ACKNOWLEDGMENTS}

We are grateful to H. Perrey (Dept. of Physics, Lund University); M. Jessen, J. Kehres, E. B. Klinkby, A. W. Larsen, M. Seelk (DTU Physics); and T. W. Hansen (DTU Nanolab) for their assistance with the work presented here. We also thank R. Jaspers, M. Messmer (Dept. of Applied Physics, Eindhoven University of Technology), and B. Schistad (Viborg Tekniske Gymnasium) for fruitful discussions, and the anonymous referees for helpful comments.

\section{DATA AVAILABILITY}

The data that support the findings of this study are available from the corresponding author upon reasonable request.

\section{REFERENCES}

${ }^{1}$ W. C. Elmore, J. L. Tuck, and K. M. Watson, Phys. Fluids 2, 239 (1959).

${ }^{2}$ G. H. Miley and S. Krupakar Murali, Inertial Electrostatic Confinement (IEC) Fusion-Fundamentals and Applications (Springer, New York, 2014).

${ }^{3}$ K. Yamauchi, M. Watanabe, A. Okino, T. Kohno, E. Hotta, and M. Yuura, Fusion Sci. Technol. 47, 1229 (2005).

${ }^{4}$ H. Osawa, S. Yoshimura, T. Tabata, and M. Ohnishi, in International Symposium on Discharges and Electrical Insulation in Vacuum, Matsue, Japan (2006), pp. 722-725

${ }^{5}$ Y. Taniuchi, Y. Matsumura, K. Taira, M. Utsumi, M. Chiba, T. Shirakawa, and M. Fujii, J. Nucl. Sci. Technol. 47, 626 (2010). 
${ }^{6}$ K. Yamauchi, K. Ogasawara, M. Watanabe, A. Okino, Y. Sunaga, and E. Hotta, Fusion Sci. Technol. 39, 1182 (2001).

${ }^{7}$ A. L. Wehmeyer, R. F. Radel, and G. L. Kulcinski, Fusion Sci. Technol. 47, 1260 (2005).

${ }^{8}$ B. J. Egle, J. F. Santarius, and G. L. Kulcinski, Fusion Sci. Technol. 52, 1110 (2007).

${ }^{9}$ T. A. Thorson, R. D. Durst, R. J. Fonck, and A. C. Sontag, Nucl. Fusion 38, 495 (1998).

${ }^{10}$ R. Bowden-Reid, J. Khachan, J.-P. Wulfkühler, and M. Tajmar, Phys. Plasmas 25, 112702 (2018).

"See https://mcnp.lanl.gov/ for "A general Monte Carlo N-particle (MCNP) transport code" (accessed 16 April 2020).

${ }^{12}$ See https://www.comsol.com/ for "COMSOL Multiphysics ${ }^{\circledR}$ modeling software" (accessed 16 April 2020).

${ }^{13}$ G. A. Emmert and J. F. Santarius, Phys. Plasmas 17, 013502 (2010).

${ }^{14}$ G. H. Miley, Y. Gu, J. M. DeMora, R. A. Stubbers, T. A. Hochberg, J. H. Nadler, and R. A. Anderl, IEEE Trans. Plasma Sci. 25, 733 (1997).

${ }^{15} \mathrm{I}$. Langmuir and K. B. Blodgett, Phys. Rev. 24, 49 (1924).

${ }^{16}$ T. Hardiment and M. D. Bowden, Phys. Plasmas 27, 043506 (2020).

${ }^{17}$ S. Krupakar Murali, G. A. Emmert, J. F. Santarius, and G. L. Kulcinski, Phys. Plasmas 17, 102701 (2010).

${ }^{18}$ R. L. Hirsch, Phys. Fluids 11, 2486 (1968).

${ }^{19}$ T. A. Thorson, R. D. Durst, R. J. Fonck, and L. P. Wainwright, Phys. Plasmas 4, 4 (1997).

${ }^{20}$ J. Khachan and S. Collis, Phys. Plasmas 8, 1299 (2001).
${ }^{21}$ A. S. Richardson, NRL Plasma Formulary (U.S. Naval Research Laboratory, Washington, 2019).

${ }^{22}$ A. N. Curren, K. A. Jensen, and G. A. Blackford, https://ntrs.nasa.gov/archive/ nasa/casi.ntrs.nasa.gov/19890008279.pdf for "Secondary electron emission characteristics of untreated and ion-textured titanium," NASA Technical Paper No. 2902, 1989.

${ }^{23}$ J. Barnard, I. Bojko, and N. Hilleret, https://arxiv.org/ftp/arxiv/papers/1302/ 1302.2333.pdf for "Measurements of the secondary electron emission of some insulators," Vacuum Technical Note 97, LHC-VAC/Io.B, 1997.

${ }^{24}$ G. H. Miley, J. M. DeMora, B. E. Jurczyk, and M. Nieto, in 18th IEEE/NPSS Symposium on Fusion Engineering. Symposium Proceedings (Cat. No. 99CH37050), Albuquerque, NM (1999), pp. 23-26.

${ }^{25}$ S. Krupakar Murali, G. L. Kulcinski, and J. F. Santarius, Phys. Plasmas 15, 122702 (2008).

${ }^{26}$ J. M. Boone and J. A. Seibert, Med. Phys. 24, 1661 (1997).

${ }^{27}$ C. R. Crowell, Solid-State Electron. 8, 395 (1965).

${ }^{28}$ D. C. Donovan, D. R. Boris, G. L. Kulcinski, J. F. Santarius, and G. R. Piefer, Rev. Sci. Inst. 84, 033501 (2013).

${ }^{29}$ G. W. McClure, Phys. Rev. 140, A769 (1965).

${ }^{30}$ S. Krupakar Murali, B. B. Cipiti, J. F. Santarius, and G. L. Kulcinski, Phys. Plasmas 13, 053111 (2006).

${ }^{31}$ H. Matsuura, T. Takaki, K. Funakoshi, Y. Nakao, and K. Kudo, Nucl. Fusion 40, 1951 (2000).

${ }^{32}$ M. Bakr, K. Masuda, and M. Yoshida, Fusion Sci. Technol. 75, 479 (2019).

${ }^{33}$ J. Hermens, R. Jaspers, and J. Khachan, Phys. Plasmas 26, 102703 (2019). 